Eurasian Journal of Physics and Functional Materials

2021, 5(2), 70-87

\title{
Synthesis reactions of neutron-rich isotopes of light elements
}

\author{
Yu.E. Penionzhkevich \\ Joint Institute for Nuclear Research, Dubna, Russia \\ National Research Nuclear University MEPhI, Moscow, Russia \\ E-mail: pyuer@mail.ru
}

DOI: 10.32523/ejpfm.2021050201

Received: 22.03.2021 - after revision

\begin{abstract}
One of the most complicated problems of experimental nuclear physics is the synthesis of exotic nuclei near the boundaries of stability. These nuclei, as a rule, are 10 or more neutrons away from stable nuclei, have a short lifetime (less than $1 \mathrm{~ms}$ ) and low binding energy. All this determines special requirements to the choice of reactions for the synthesis of such nuclei and the method of their transportation and registration. Mainly, for the synthesis of exotic nuclei, reactions of fragmentation of the bombarding heavy ion, direct reactions of the types $(p, d),(d, p),(d, n),\left(d,{ }^{3} \mathrm{He}\right)$, etc., as well as reactions of fission and deep inelastic transfer are used.
\end{abstract}

Keywords: neutron-rich isotopes, nuclear reactions, exotic nuclei, beams of radioactive nuclei.

\section{Introduction}

As will be seen from the following, it is now possible to obtain information on nuclei with a large excess of neutrons $(N / Z / A)$ only by studying light nuclei. Only in the region of the lightest nuclei we can reach the boundary of neutron stability and observe nuclei that decay by emitting neutrons. Interest in neutronrich nuclei with is caused by many factors, but primarily by the fact that the position of the boundary of neutron stability, and even its existence, is, in essence, an open question. Despite numerous calculations of the masses of nuclei, they are all not sufficiently reliable when moving away from the stability valley. The question of the possibility of the existence of nuclei in which the neutron excess is much larger than the predicted one has been repeatedly discussed in the literature, 
and in the limiting case, the existence of nuclei consisting of neutrons alone is not excluded. If the possibility of the existence of nuclei with an anomalously large neutron excess (we will call them neutron nuclei) was demonstrated, it would be a qualitative change in our present ideas about the nucleus. This would have an undoubted influence on other sciences, for example, astrophysics [1]. Extrapolation of the properties of nuclei near the line of $\beta$-stability, as a rule, does not provide information on what can be expected for nuclei far from the stability region. Therefore, direct experiments for studying the structure of light nuclei at the boundary of nucleon stability are of great importance for understanding the properties of such nuclei and predicting the properties of heavier nuclei. The new possibilities for these experiments appeared with obtaining sufficiently intense (up to $10^{8} \mathrm{~s}^{-1}$ ) beams of radioactive nuclei. One of the most complicated problems of experimental nuclear physics is the synthesis of exotic nuclei near the boundaries of stability. These nuclei, as a rule, are 10 or more neutrons away from stable nuclei, have a short lifetime (less than $1 \mathrm{~ms}$ ) and low binding energy. All this determines special requirements to the choice of reactions for the synthesis of such nuclei and the method of their transportation and registration. Mainly, for the synthesis of exotic nuclei, reactions of fragmentation of the bombarding heavy ion, direct reactions of the types $(p, d),(d, p),(d, n),\left(d,{ }^{3} \mathrm{He}\right)$ etc., as well as reactions of fission and deep inelastic transfer are used [1].

\section{Fission reactions}

Spontaneous fission of nuclei is accompanied by the emission of light charged particles formed at the moment of scission of the heavy nucleus into two fragments. There is a high probability that these light charged particles are neutron-rich. During the spontaneous fission of ${ }^{252} \mathrm{Cf}$, neutron-rich isotopes of hydrogen, helium, lithium, beryllium, and even heavier elements were observed [2]. The search for such exotic nuclei as ${ }^{10} \mathrm{He}$ and ${ }^{7} \mathrm{H}$ was also carried out in fragments of spontaneous fission of ${ }^{252} \mathrm{Cf}$ [3]. However, in these experiments there were no events that could be attributed to the ${ }^{10} \mathrm{He}$ nucleus. The upper limit for the probability of fission with the emission of ${ }^{10} \mathrm{He}$ with respect to the emission of ${ }^{8} \mathrm{He}$ was $Y\left({ }^{10} \mathrm{He}\right) / Y\left({ }^{8} \mathrm{He}\right)<4 \times 10^{-3}$. At the same time, in [3] the authors believe that several events corresponding to the ${ }^{10} \mathrm{He}$ nucleus were observed. Experiment [4] yielded the ratio $Y\left({ }^{7} \mathrm{H}\right) / Y\left({ }^{3} \mathrm{H}\right)<10^{-4}$ for ${ }^{7} \mathrm{H}$.

The fission by thermal neutrons was used to synthesize exotic neutron-rich nuclei with $A=60-160$ [5]. Experiments on the study of fission induced by ${ }^{40} \mathrm{Ca}$ and ${ }^{48} \mathrm{Ca}$ ions [6] showed that a strong neutron excess of the bombarding ion can lead to the synthesis of nuclei located farther from the line of $\beta$-stability. It was also shown that fission induced by protons [7] and especially by heavier ions [8] leads to an increase of the probability of triple fission with the emission of light particles and nuclei. This is a consequence of a higher excitation energy and a higher angular momentum of the fissioning nucleus. However, the high excitation energy transferred to the fragments forces them to evaporate neutrons and thereby reduces the probability of formation of neutron-rich nuclei. 


\section{Reactions of fragmentation of target nucleus}

The reactions of fragmentation of a target nucleus are efficient for the synthesis of neutron-rich nuclei if as a bombarding particle protons with energy from several $\mathrm{GeV}$ and higher and targets with $\mathrm{Z}>80$ are used. The position of the maximum of the isotope distribution of products depends on the mass of the target. With an increase in the $N / Z$ ratio for the target, the $N / Z$ value for the products of the fragmentation reaction also increases.

During the 1960-1970's, the availability of high-energy proton accelerators made it possible to use these reactions for the synthesis of new nuclei. For example, at that time ${ }^{11} \mathrm{Li},{ }^{14} \mathrm{~B},{ }^{15} \mathrm{~B},{ }^{17} \mathrm{C},{ }^{19} \mathrm{C},{ }^{19} \mathrm{~N},{ }^{21} \mathrm{O}$ were synthesized for the first time $[9,10]$. Based on the systematics [11] of the cross sections for the formation of neutron-rich nuclei in the reactions of fragmentation of the target nucleus, it can be concluded that the probability for the formation of nuclei with extreme values of neutrons excess is very high. In [12] estimates of the cross sections for the formation of several nuclear systems are given: $\approx 10^{-30} \mathrm{~cm}^{2}$ for ${ }^{8} n, \approx 10^{-29} \mathrm{~cm}^{2}$ for ${ }^{10} \mathrm{He}$, and $\approx 10^{-30} \mathrm{~cm}^{2}$ for ${ }^{13} \mathrm{Li}$. Recently, another method is discussed for obtaining exotic nuclei in quasi-fission reactions [13], when two interacting heavy nuclei form a dinuclear system which, not reaching an equilibrium configuration, separates into fragments directly from the saddle point [14]. The quasi-fission reaction products have a wide variation of mass and charge. It is possible that these reactions may prove to be efficient for the synthesis of heavy nuclei. With the advent of intense heavy-ion beams, reactions with accelerated heavy ions become the most efficient method of obtaining nuclei at the boundaries of nucleon stability.

\section{Reactions of fragmentation of bombarding ion}

This type of reactions has been used for synthesizing and studying the properties of exotic nuclei with the advent of the possibility of accelerating heavy ions up to energies $E>40 \mathrm{MeV} / \mathrm{A}$. Early experiments using ${ }^{12} \mathrm{C},{ }^{14} \mathrm{~N},{ }^{16} \mathrm{O}$, and ${ }^{20} \mathrm{Ne}$ beams at energies of $1-2 \mathrm{GeV} / \mathrm{A}$ have shown that relativistic collisions between complex nuclei lead to the formation of nuclei far from the line of stability [15]. The first works on the synthesis of new nuclei were performed using ${ }^{40} \mathrm{Ar}$ and ${ }^{48} \mathrm{Ca}$ beams with an energy of $\approx 200 \mathrm{MeV} / \mathrm{A}$, and at that time about 15 new exotic nuclei were synthesized from nitrogen to chlorine [16].

Fragmentation reactions have characteristics that lead to high efficiency of the synthesis of new exotic nuclei. Firstly, it is possible to obtain reaction products in a wide range of $A$ and $Z$. Secondly, the velocities of reaction products practically coincide with the velocity of the incident ion, which allows one to use them without subsequent acceleration and to separate them from other reaction products by means of the same magnetic optics as for the primary beam. Thirdly, because of the large transport velocity, the reaction products have angular distributions that are strongly directed forward, which substantially increases the geometric efficiency of their collection. Finally, the $N / Z$ ratio in reaction 
products is sensitive to the ratio of the number of neutrons and protons in the bombarding ion. It was experimentally shown that the yield, for example, of neutron-rich sodium isotopes in the reaction with ${ }^{48} \mathrm{Ca}$ ions is much higher than in the reaction with ${ }^{40} \mathrm{Ar}$ ions [17]. Thus, for the synthesis of neutron-rich nuclei in the reactions of fragmentation of the bombarding ion, it is preferable to use beams of such nuclei as ${ }^{36} \mathrm{~S},{ }^{48} \mathrm{Ca},{ }^{58} \mathrm{Fe}$, etc. The fact that the isotopic distributions of reaction products strongly correlate with the $N / Z$ ratio in the bombarding ion is also confirmed by the calculations [18]. In work [19], new isotopes ${ }^{27,29,31} \mathrm{~F}$ were synthesized using the ${ }^{48} \mathrm{Ca}$ beam. Using the ${ }^{56} \mathrm{Fe}$ beam (670 MeV/A), the heaviest boron isotope ${ }^{19} \mathrm{~B}$ was observed [20]. This isotope was obtained as a result of 'stripping' of 37 nucleons (21 protons and 16 neutrons) from the bombarding ion. In the joint experiments of Dubna and GANIL (France) in the fragmentation reactions of ${ }^{40} \mathrm{Ar},{ }^{48} \mathrm{Ca}$, and ${ }^{86} \mathrm{Kr}$ beams at intermediate energies $(E>35 \mathrm{MeV} / \mathrm{A})$, a large number of neutron-rich isotopes of carbon, nitrogen, fluorine, neon, argon, titanium, vanadium, chromium, manganese, iron, and cobalt was obtained [21].

In Japan (RIKEN) using a ${ }^{50} \mathrm{Ti}$ beam energy of $80 \mathrm{MeV} / \mathrm{A}$, two new neutronrich isotopes ${ }^{31} \mathrm{Ne}$ and ${ }^{37} \mathrm{Mg}$ were synthesized [22], and in the joint DubnaRIKEN experiment with a ${ }^{48} \mathrm{Ca}$ beam at an energy of $70 \mathrm{MeV} / \mathrm{A}$ for the first time new isotopes ${ }^{38} \mathrm{Mg}$ and ${ }^{40,41} \mathrm{~A} 1$ [23] were observed. Finally, in the ${ }^{48} \mathrm{Ca}$ fragmentation reaction on the accelerator of the University of Michigan, the last stable magnesium isotope, ${ }^{40} \mathrm{Mg}$, was obtained [24]. All these experiments convincingly showed that the use of beams of neutron-rich nuclei makes it possible to obtain new isotopes in the reactions of fragmentation of the bombarding ion with yields several orders of magnitude higher than the corresponding yields in other reactions. Such characteristics of reactions of fragmentation of bombarding ions, as a narrow forward-directed angular distribution of products with a momentum close to the beam momentum, are efficiently used in producing beams of radioactive nuclei. Thus, it is possible to "collect" the resulting products and form from them secondary beams of radioactive nuclei. This new direction in nuclear physics, which has been intensively developing recently, made it possible to obtain unique information on the structure of exotic nuclei by measuring such experimental quantities as reaction cross sections, momentum distributions, and correlations between the products of the breakup of the investigated nuclei. With the use of beams of radioactive nuclei, important discoveries have been made, such as the existence of a neutron halo in ${ }^{11} \mathrm{Li}$ [25], the observation of ${ }^{10} \mathrm{He}$ in the reaction with the ${ }^{11} \mathrm{Li}$ beam [26], etc. Thus, large prospects in the study of exotic nuclei open due to the use of secondary beams of radioactive nuclei [27].

Let us briefly describe the main characteristics of the fragmentation reactions and the possibility of using them for the synthesis of nuclei near the shells $N=20$ and $N=28$. Usually, fragmentation reactions yield nuclei with $Z$ and $A$ smaller than the $Z$ and $A$ of the bombarding heavy ion. Meanwhile, as shown in [18], statistical methods of calculations can be used to describe the fragmentation reactions. A proof of the statistical nature of fragmentation is the effect of "memory" of the beam: for more neutron-rich or neutron-deficient beams, fragments "remember" the excess of neutrons or protons of the beam. Therefore, 
for the synthesis of neutron-rich light nuclei near the shells $N=20$ and $N=28$, the beams of rare neutron-rich isotopes such as ${ }^{36} \mathrm{~S}(N=20)$ or ${ }^{48} \mathrm{Ca}(N=28)$ are the most optimal.

Let us give the main expressions for estimating the yields of secondary particles obtained in the fragmentation reaction. The main factors influencing the yield of the nuclei are the choice of the primary beam, the influence of its energy on the yield, and the choice of the optimal thickness of the target taking into account the characteristics of the fragment separator used. For fragmentation reactions, the correct choice of the optimal thickness of the target is necessary. Specific losses of the ion $(A, Z)$ during the passage through the target are expressed by the formula

$$
\Delta E \approx \frac{A Z^{2}}{E}
$$

The uncertainty in the momentum distribution of the fragment after the passage through the target can be determined from two boundary conditions: the reaction occurred at the beginning $(i)$ of the target and the reaction occurred at the end $(f)$ of the target, respectively:

$$
\frac{\Delta p}{p} \approx t \cdot\left(\frac{\Delta E_{i}}{E_{i}}-\frac{\Delta E_{i}}{E_{i}}\right) \approx t \cdot\left(Z_{i}^{2}-Z_{f}^{2}\right) / E^{2},
$$

where $t$ is the thickness of the target, and $Z_{i, f}$ are the charges of the fragments at different locations in the target.

It is assumed that the velocity of the fragment is close to the velocity of the bombarding particle. The maximum yield of secondary products for the optimal thickness $t$ of the target is achieved if the losses in the transmission due to the broadening of the momentum distribution are greater than the increase in the yield of the products due to the thickness of the target.

The yield $Y_{S}$ of fragments is proportional to the thickness of the target as well as angular and momentum variance of the beam. Taking into account the above dependence, we obtain the following formula for the yield of fragments:

$$
Y_{s} \approx t_{o p t} /\left(\sigma_{\theta} \cdot \sigma_{\phi} \cdot \sigma_{\frac{\Delta p}{p}}\right) \approx E_{i}^{2} \cdot E_{i}^{0.5} \cdot E_{i}^{0.5} \cdot E_{i}^{0.5}=E_{i}^{7 / 2},
$$

We see that the intensity of the secondary beam depends on the energy of the primary beam as $E^{7 / 2}$. As shown in [28], this formula is applicable for primary beam energy values up to $100 \mathrm{MeV} / \mathrm{A}$. At large values of energy, the yield of fragments decreases because the variance of the momentum distribution becomes much smaller than the momentum capture of the fragment separators used. When choosing $A$ and $Z$ of the primary beam, it must be borne in mind that the number of fragments should be proportional to $Z$. Taking into account the energy losses $\Delta E / E \approx A Z^{2} /(E E) \approx Z^{2}$, we can finally obtain the dependence of the yield of fragments on the beam charge $\approx 1 / Z^{3}$. Thus, it is obvious that in order to obtain the maximum yield of fragments, the primary beam must be as light as possible.

For an approximate estimation of the cross section, one can use the simplified expression 


$$
\sigma=\exp \left(-0.1763 Z_{\text {beam }}\right)
$$

At present, the LISE program is used to calculate the yields of nuclei at the boundaries of nucleon stability [18], which in most cases gives a satisfactory description of the experimental results. Despite the fact that this estimate of the intensity of the secondary beam is rather rough, it allows us to conclude that the energy of the primary beam is not the main factor for increasing the yield of neutron-rich isotopes of light nuclei. This conclusion is applicable only for obtaining nuclei by fragmentation.

Thus, in order to obtain neutron-rich isotopes, it is better to use a primary beam of neutron-rich nuclei with the atomic number closest to the product being synthesized.

\section{Reactions with heavy ions accompanied by emission of fast particles}

These reactions in the late 1970s and 1980s attracted the attention of physicists by the unusual mechanism of formation of fast particles.

As early as 1961, two components in the distribution of particles, formed in reactions with heavy ions, were observed in [29]. One of them was evaporation $\alpha$-particles, the second component was a high-energy one, with strongly-forward angular distribution and with a maximum yield at energy corresponding to the velocity of the bombarding ion.

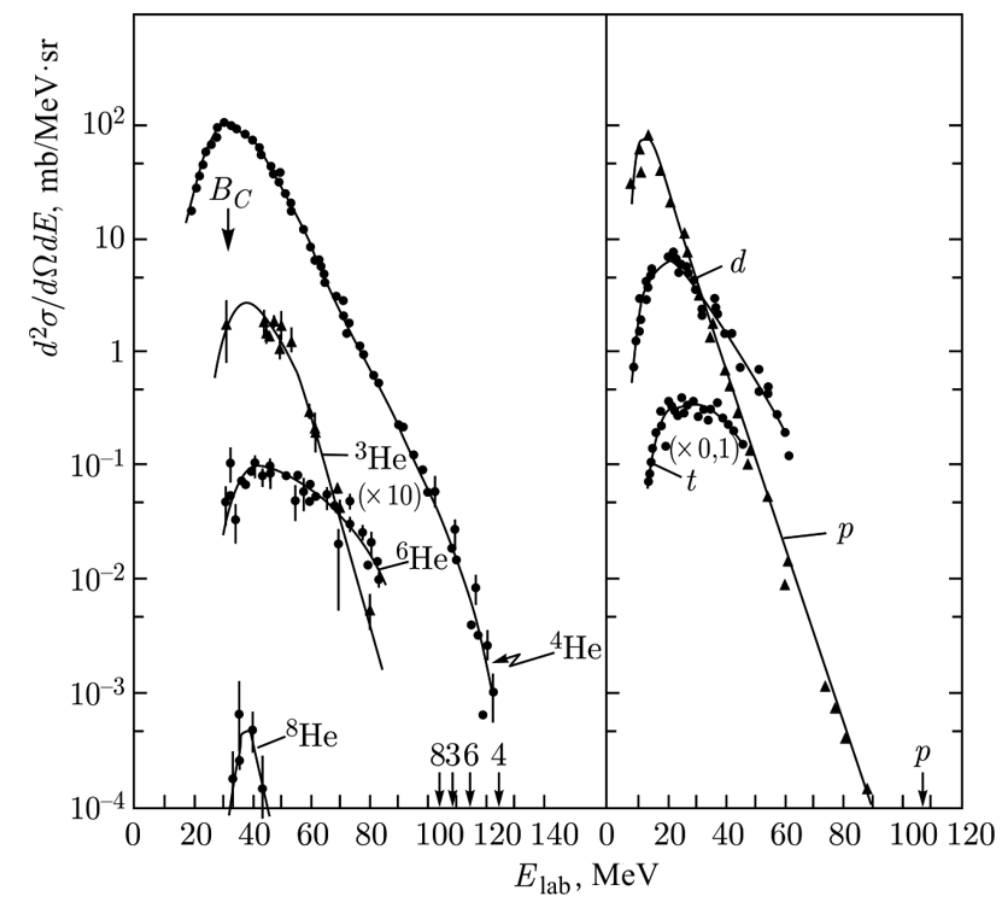

Figure 1. The energy spectra of hydrogen and helium isotopes measured in the reaction ${ }^{232} \mathrm{Th}+{ }^{22} \mathrm{Ne}(178 \mathrm{MeV})$ at the angle of $0^{\circ}$. The arrow at $B_{c}$ denotes the exit Coulomb barrier. The arrows on the $E_{l a b}$ axis denote kinematic limits for different particles [30]. 
Further experiments showed that in reactions with ions heavier than ${ }^{12} \mathrm{C}$, the emission of heavier charged particles occurs with a noticeable cross section [30].

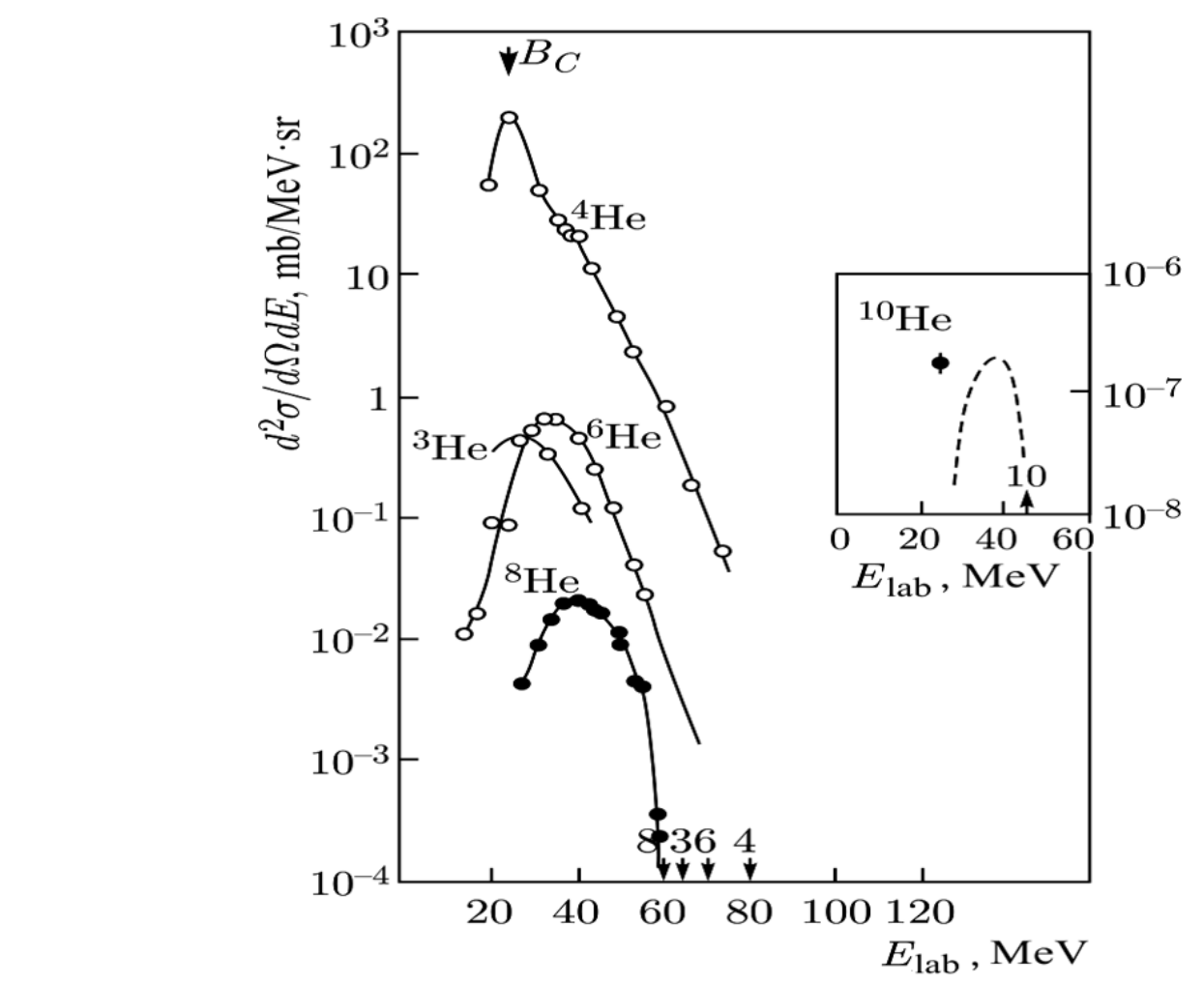

Figure 2. Energy spectra of helium isotopes measured in the reaction ${ }^{232} \mathrm{Th}+{ }^{11} \mathrm{~B}(89 \mathrm{MeV})$ at the angle of $20^{\circ}$. The inset shows the expected shape of the energy spectrum of the ${ }^{10}$ He nucleus [31].

The phenomenological model, based on the assumption that part of the incident ion is transferred to the target nucleus, and the rest flies forward, reproduces the isotope formation sections with $Z=1-4$ quite well. At the same time, other characteristics of the process could not be explained within the framework of the same model [32]. From Figure 2 it can be seen that the energy spectra of different particles exponentially decrease with increasing energy of the particles until the moment when this energy is only several $\mathrm{MeV}$ different from the maximum possible value allowed by the energy and momentum conservation laws in the case of a two-body reaction mechanism [30].

When the energy of the particles is equal to the energy at the kinematic limit, the two nuclei formed in the exit channel are in the ground states. The difference observed between the experimental and calculated values of the limit energy is determined by the angular momentum of the residual heavy nucleus, which depends on the energy of the bombarding ion and on the type of the emitted particle. For example, in the case of the emission of beryllium nuclei, their energy reaches a kinematic limit with a relatively large cross section $\left(\approx 10^{-30}\right.$ $\left.\mathrm{cm}^{2} /(\mathrm{MeV} \times \mathrm{sr})\right)$. Based on the observed effect, it can be assumed that reactions with the emission of fast particles can be used for the synthesis of exotic nuclei. In these reactions, an attempt was made to synthesize such exotic nuclei as ${ }^{10} \mathrm{He}$, ${ }^{14} \mathrm{Be}$, and others. 


\section{Charge-exchange reactions}

In charge-exchange reactions, one or several protons are replaced by one or several neutrons. The simplest reactions of this type are $(p, n)$ or $(n, p)$. In this case, although the charge of the nucleus changes, its mass number is conserved.

Reactions of double charge exchange induced by $\pi$-mesons $\left(\pi^{-}, \pi^{+}\right)$occupy a special place in the search for exotic nuclei, such as $4 n,{ }^{5} \mathrm{H},{ }^{6} \mathrm{H},{ }^{7} \mathrm{H},{ }^{9} \mathrm{He}$ [33]. In the reaction ${ }^{11} \mathrm{~B}\left(\pi^{-}, \pi^{+}\right){ }^{11} \mathrm{Li}$, the mass of ${ }^{11} \mathrm{Li}$ was determined with great accuracy and an excited level was observed at the energy $E^{*}=(1.2 \pm 0.1)$ $\mathrm{MeV}$, which was interpreted as the observation of a soft dipole resonance in this nucleus [34]. Measurement of the mass of the ${ }^{9} \mathrm{He}$ nucleus in the reaction with $\pi$-mesons [35] made it possible to conclude that the ${ }^{10} \mathrm{He}$ nucleus may prove to be more stable than predicted by different models, and thus this was a stimulating factor for its further search. When heavy ions are used, chargeexchange reactions also take place with a large cross section. The first studies using double charge exchange with heavy ions [36] showed that the cross section of the reaction increases with the energy of the bombarding ion and that the angular distributions are forward directed. In addition, it was shown in [37] that nuclei in the ground states are formed in the charge-exchange reactions. Figure 3 shows the systematics of the known cross sections for single and double chargeexchange reactions leading to the formation of final nuclei in the ground states. It can be concluded that these cross sections correlate with the $Q$-value of the reaction. The experimental points up to the reaction $Q$-values of about $-20 \mathrm{MeV}$ were obtained at beam energies up to $10-15 \mathrm{MeV} / \mathrm{A}$, the results for more negative $Q$-values were obtained at beam energies about 25-35 MeV/A. Apparently, the cross section of the charge-exchange reaction depends also on the energy of the bombarding ion, beside the reaction $Q$-value. Such a conclusion was made in [36], in which the question of the influence of the energy of the bombarding ion on the mechanism of the charge-exchange reaction was studied: is there a direct charge exchange or a sequential exchange of proton(s) for neutron(s).

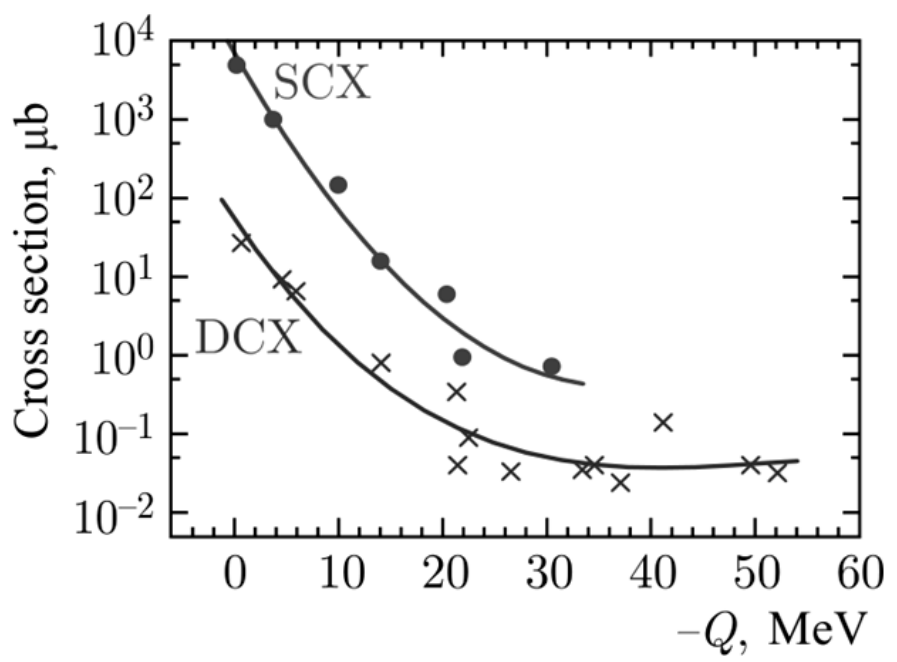

Figure 3. The systematics of the known cross sections for single (SCX) and double (DCX) charge-exchange reactions obtained with beams of heavy ions leading to the formation of final nuclei in the ground states depending on the reaction $Q$-value. 
The mechanism of such a reaction depends not only on the energy, but also on what levels are populated, that is, the structure of interacting nuclei also has a significant influence. In work [38], on the example of reactions $\left({ }^{12} \mathrm{C},{ }^{12} \mathrm{~N}\right)$, $\left({ }^{12} \mathrm{C},{ }^{12} \mathrm{~B}\right),\left({ }^{13} \mathrm{C},{ }^{13} \mathrm{~N}\right),\left({ }^{13} \mathrm{C},{ }^{13} \mathrm{~B}\right)$, high selectivity of charge-exchange reactions was shown. Choosing a suitable combination of a target and a bombarding ion, one can achieve the population of different final states with a greater or lower cross-section value.

For example, in the reaction $\left({ }^{12} \mathrm{C},{ }^{12} \mathrm{~N}\right)$ the population of states with $\Delta S=1$ dominates, i.e., with an unnatural parity, whereas in the reaction $\left({ }^{13} \mathrm{C},{ }^{13} \mathrm{~N}\right)$, where $\Delta S=1$, non-spin-flip transitions dominate, while in the reaction $\left({ }^{13} \mathrm{C},{ }^{13} \mathrm{~B}\right)$ both types of level population take place, however, the transitions with $\Delta S=1$ are more probable (Figure 4).

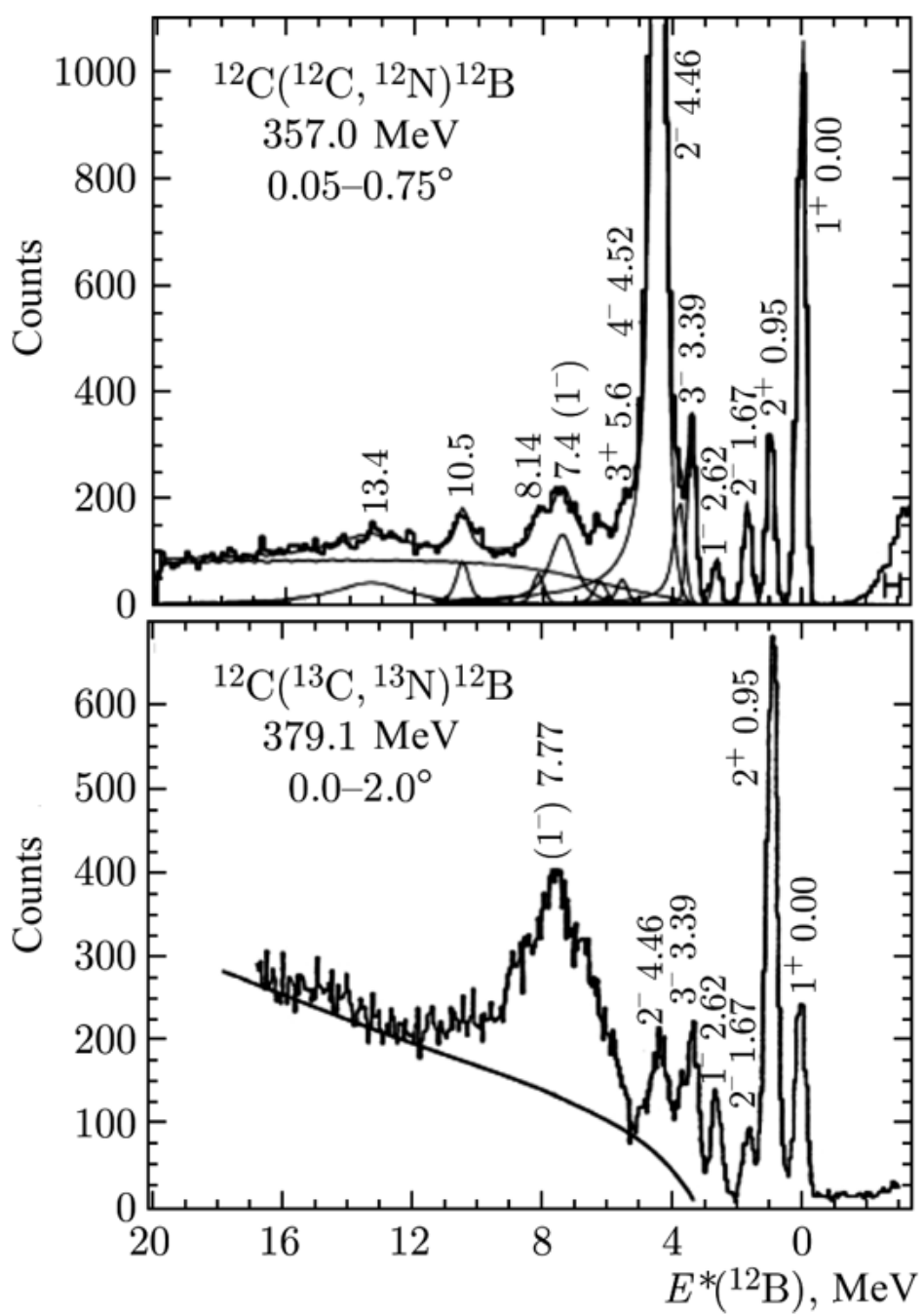

Figure 4. The spectra of ${ }^{12} \mathrm{~N}$ and ${ }^{13} \mathrm{~N}$ showing the different probabilities of population of the ${ }^{12} \mathrm{~B}$ levels in the reactions ${ }^{12} \mathrm{C}\left({ }^{12} \mathrm{C},{ }^{12} \mathrm{~N}\right){ }^{12} \mathrm{~B}$ and ${ }^{12} \mathrm{C}\left({ }^{13} \mathrm{C},{ }^{13} \mathrm{~N}\right){ }^{12} \mathrm{~B}$ on the target $0^{+}$spin as a result of the transitions $0^{+} \rightarrow 1^{+}$ and $1 / 2^{-+} \rightarrow 1 / 2^{-}$in the bombarding nucleus. It is seen that in the reaction ${ }^{12} \mathrm{C}\left({ }^{12} \mathrm{C},{ }^{12} \mathrm{~N}\right){ }^{12} \mathrm{~B}$, unnatural parity states are more strongly manifested than those of their multiplet counterparts [38].

Due to the fact that the cross sections of the charge-exchange reactions with heavy ions turned out to be relatively large and these reactions are optimal for the population of individual levels, it was concluded that they can be used for the synthesis of nuclei near the nucleon stability boundary. For example, in the 
reactions ${ }^{9} \mathrm{Be}\left({ }^{13} \mathrm{C},{ }^{13} \mathrm{O}\right){ }^{9} \mathrm{He}$ and ${ }^{9} \mathrm{Be}\left({ }^{14} \mathrm{C},{ }^{14} \mathrm{O}\right){ }^{9} \mathrm{He}$, the ${ }^{9} \mathrm{He}$ nucleus was studied [39]. The obtained cross section for the production of ${ }^{9} \mathrm{He}$ in the ground state was $\approx 40 \mathrm{nb} / \mathrm{sr}$. In [40], the ${ }^{13}$ Be nucleus was studied in the reaction $\left({ }^{14} \mathrm{C}\right.$, ${ }^{14} \mathrm{O}$ ) on the ${ }^{13} \mathrm{C}$ target, and ${ }^{14} \mathrm{C}\left({ }^{11} \mathrm{~B},{ }^{11} \mathrm{Li}\right)$ reaction was used to study the ${ }^{11} \mathrm{Li}$ nucleus. In the double charge-exchange reaction ${ }^{10} \mathrm{Be}\left({ }^{14} \mathrm{C},{ }^{14} \mathrm{O}\right){ }^{10} \mathrm{He}$, the mass of the ${ }^{10} \mathrm{He}$ nucleus was determined with high accuracy and two excited levels were discovered [41]. The cross section for the production of ${ }^{10} \mathrm{He}$ in this reaction was $140 \mathrm{nb} / \mathrm{sr}$.

Thus, charge-exchange reactions with heavy ions are a good tool in the study of exotic nuclei. It is worth noting once again that the mechanism of these reactions under certain conditions can be considered as a special case of the mechanism of transfer reactions which will be discussed below.

\section{Features of transfer reactions for spectroscopy of light exotic nuclei}

By such reactions we mean reactions of the type $A(a, b) B$, in which protons and/or neutrons are exchanged between the target nucleus and the bombarding ion, which leads to the formation of nuclei $b$ and $B$ in the exit channel. Such transfer can proceed via several stages (sequential transfer).

A detailed review of information on light exotic nuclei in such reactions is presented in [42]. The use of these reactions shows the efficiency of this method for the study of exotic nuclei. When three-five nucleons were transferred, it became possible to reach the neutron stability boundary for nuclei with mass number $A<16$ and to discover unbound nuclei beyond this boundary in the form of resonances. For example, the nuclei ${ }^{7-10} \mathrm{He},{ }^{10,11} \mathrm{Li},{ }^{10-14} \mathrm{Be},{ }^{14-16} \mathrm{~B}$ were studied using transfer reactions with beams of ${ }^{12,13,14} \mathrm{C}, 14,15 \mathrm{~N}$, and ${ }^{16} \mathrm{O}$ at the accelerator complex of heavy ions in Berlin [43], and the ${ }^{11} \mathrm{Li}$ and ${ }^{13} \mathrm{Be}$ nuclei were studied with the ${ }^{11} \mathrm{~B}$ beam at the U400 accelerator, FLNR (Dubna), and the accelerator in NSCL (Michigan). It should be mentioned that the cross sections for the reactions of few-nucleon transfer leading to ground states of final exotic nuclei may be very small. The range of cross sections extends from several nanobarns to microbarns. It is very difficult to make an estimation of the cross sections for nuclear fusion reactions in the region of the nucleon stability boundary. However, based on the literature data, it can be assumed that the yield of the studied nuclei depends on many factors, such as the number of transferred nucleons, the reaction $Q$-value, the structure of the nuclei involved in the reaction, the detection angle, the bombarding heavy ion energy. It was also shown that the cross sections of the transfer reactions are relatively large only if certain conditions are met [44]. These conditions are related to the conservation of momentum and angular momentum in the initial and final nuclei and are determined by the following expressions:

$$
\Delta k=k_{0}-\lambda_{1} / R_{1}-\lambda_{2} / R_{2} \approx 0,
$$




$$
\lambda L=\lambda_{2}-\lambda_{1}+1 / 2 k_{0}\left(R_{1}-R_{2}\right)+Q_{e f f} R /(\hbar v) \approx 0,
$$

where $Q_{\text {eff }}$ is calculated as

$$
Q_{e f f}=Q-\left(Z_{1}^{f} Z_{2}^{f}-Z_{1}^{i} Z_{2}^{i}\right) e^{2} / R,
$$

Here $Z_{1,2}^{f, i}$ are the atomic numbers of the nuclei in the final and initial states; $R_{1,2}$ are radii of the two nuclei, $R=R_{1}+R_{2}, k_{0}=m v / \hbar$ is the momentum of the transferred nucleon; $\lambda_{1}, \lambda_{2}$ are the projections of the orbital angular momentum $l_{1}$ and $l_{2}$ of the transferred nucleon in the initial and final states; $v$ is the relative velocity of two interacting nuclei in the transfer region. The value of $Q_{e f f}$ equals the reaction $Q$-value if the transferred particle is not charged. It was shown that in the reactions with light nuclei charge correction can be neglected and $Q_{\text {eff }} \approx Q$.

The third condition requires the sums $l_{1}+\lambda_{1}$ and $l_{2}+\lambda_{2}$ to be even numbers. Deviations from these conditions lead to a decrease in the cross section for the reactions of nucleon transfer to particular final states. The mechanism of the reactions of one-, two-, three-, etc. nucleon transfer was considered in detail in [45]. The systematics of the cross sections for the formation of nuclei in the ground states in the reactions of transfer of a different number of nucleons as a function of the reaction $Q$-value was obtained in [46].

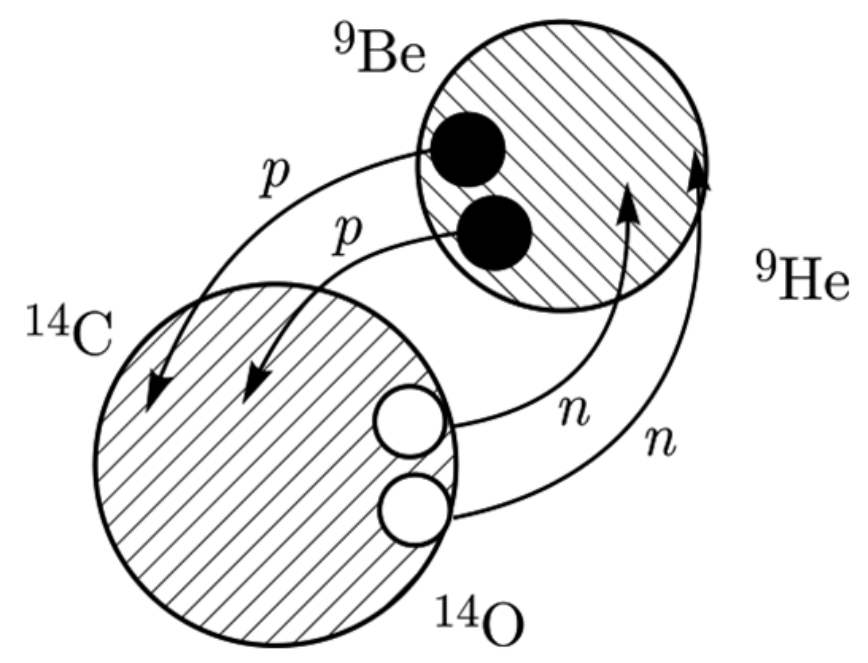

Figure 5. The mechanism of transfer in the double charge-exchange reaction ${ }^{9} \mathrm{Be}\left({ }^{14} \mathrm{C},{ }^{14} \mathrm{O}\right){ }^{9} \mathrm{He}$.

Let us try to give concrete examples of the reactions that proceed with the pickup of protons and the stripping of neutrons. Figure 5 schematically shows the mechanism of this transfer in the reaction ${ }^{9} \mathrm{Be}\left({ }^{14} \mathrm{C},{ }^{14} \mathrm{O}\right){ }^{9} \mathrm{He}$ : two neutrons are transferred to the target from by bombarding ion (the first stage) and two protons are transferred in the opposite direction (second stage) or vice versa.

As a result, the following processes take place for the reaction ${ }^{9} \mathrm{Be}\left({ }^{14} \mathrm{C}\right.$, $\left.{ }^{14} \mathrm{O}\right){ }^{9} \mathrm{He}$ :

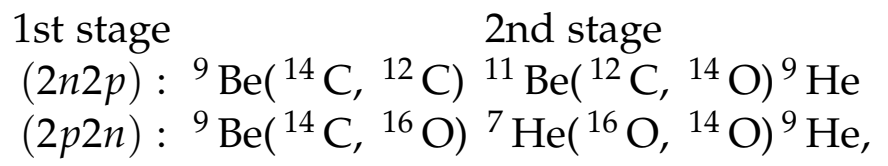


or for the reaction ${ }^{9} \mathrm{Be}\left({ }^{13} \mathrm{C},{ }^{13} \mathrm{O}\right){ }^{9} \mathrm{He}$ :

$\begin{array}{ll}\text { 1st stage } & \text { 2nd stage } \\ (2 n 2 p):{ }^{9} \mathrm{Be}\left({ }^{13} \mathrm{C},{ }^{11} \mathrm{C}\right){ }^{11} \mathrm{Be}\left({ }^{11} \mathrm{C},{ }^{13} \mathrm{O}\right){ }^{9} \mathrm{He} \\ (2 p 2 n):{ }^{9} \mathrm{Be}\left({ }^{13} \mathrm{C},{ }^{15} \mathrm{O}\right){ }^{7} \mathrm{He}\left({ }^{15} \mathrm{O},{ }^{13} \mathrm{O}\right){ }^{9} \mathrm{He} .\end{array}$

In addition to the two-step processes, one can take into account the contribution from three- and four-step processes by considering the transfer of individual nucleons, which, for example, proceed via the following stages:

$$
\begin{aligned}
& { }^{9} \mathrm{Be}\left({ }^{13} \mathrm{C},{ }^{12} \mathrm{C}\right){ }^{10} \mathrm{Be}\left({ }^{12} \mathrm{C},{ }^{11} \mathrm{C}\right){ }^{11} \mathrm{Be}\left({ }^{11} \mathrm{C},{ }^{13} \mathrm{O}\right){ }^{9} \mathrm{He} \text { or } \\
& { }^{9} \mathrm{Be}\left({ }^{13} \mathrm{C},{ }^{14} \mathrm{~N}\right){ }^{8} \mathrm{Li}\left({ }^{14} \mathrm{~N},{ }^{15} \mathrm{O}\right){ }^{7} \mathrm{He}\left({ }^{15} \mathrm{O},{ }^{13} \mathrm{O}\right){ }^{9} \mathrm{He} .
\end{aligned}
$$

In this case, as shown in Figure 6, beside two-stage amplitudes, there are six three- and six four-stage amplitudes.

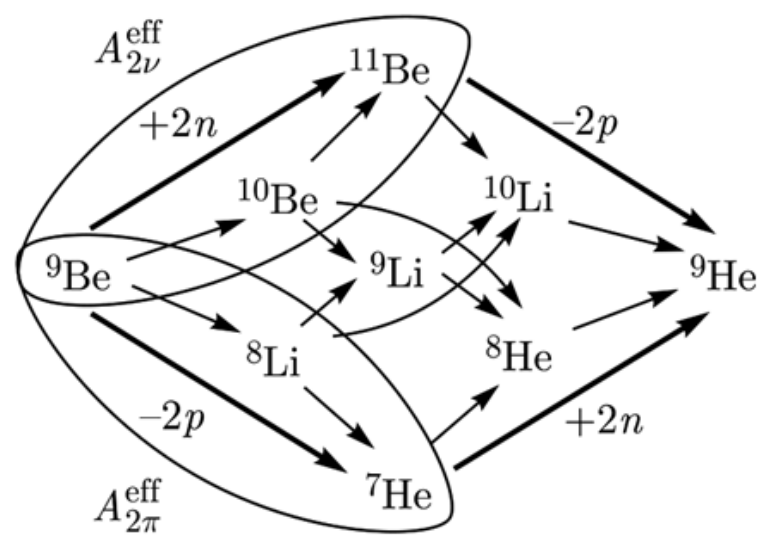

Figure 6. Multistage contributions for transitions in the target nucleus leading to the ${ }^{9}$ He nucleus. The contributions to the effective spectroscopic amplitudes $A_{2 v}^{e f f}$ and $A_{2 \pi}^{e f f}$ of the first stage are surrounded by a line.

However, the contribution of these processes is a small fraction of the total cross section. We can assume that the measured spectroscopic amplitudes for neutron and proton pairs actually include the contributions of higher-order amplitudes, such as those for transfer of individual nucleons and inelastic scattering. Consequently, the product of spectroscopic amplitudes obtained in the experiment is an effective spectroscopic amplitude $A_{2 v}^{\text {eff }}=A_{2 v}^{e f f, t a r g} \times A_{2 v}^{e f f, p r o j}$ (similarly for $A_{2 \pi}^{e f f}$ ).

Two-stage calculations are usually carried out with the FRESCO code [47] within the coupled-channel model using the same $A^{\text {eff }}$ values for the second stage as those used for the first stage. As for the reaction ${ }^{9} \mathrm{Be}\left({ }^{13} \mathrm{C},{ }^{11} \mathrm{C}\right){ }^{11} \mathrm{Be}$, the reaction cross sections corresponding to the first stage were determined experimentally.

Thus, the method using effective spectroscopic amplitudes measured for individual transfer stages works successfully in the case of $2 n 2 p$ - and $2 p 2 n$ exchange. Therefore, based on the measured cross sections for the reactions of transfer of one and two nucleons, it is possible to predict the cross sections for the transfer reactions of three and four nucleons with a sensitivity of several $\mathrm{nb} / \mathrm{sr}$, which is used in estimating the possibilities of such experiments. 


\section{Deep inelastic transfer reactions, quasi-fission}

In the late 1960's the use of transfer reactions with heavy ions ${ }^{11} \mathrm{~B},{ }^{15} \mathrm{~N},{ }^{18} \mathrm{O}$, ${ }^{22} \mathrm{Ne}$, and ${ }^{40}$ Ar made it possible to synthesize about 30 new isotopes of carbon, nitrogen, oxygen, fluorine, neon, magnesium, aluminum, silicon, phosphorus, sulfur and chlorine [48]. Much later, the same mechanism using beams of ${ }^{40} \mathrm{Ar}$ and ${ }^{56} \mathrm{Fe}$ was used to obtain even more neutron-rich isotopes of elements with $Z=14-26[49,50]$.

The first information that in the interaction of two complex nuclei a massive transfer of nucleons can occur, accompanied by a significant loss of kinetic energy, was obtained in Dubna in the late 1960's [51].

Further experimental and theoretical studies in this direction revealed certain regularities in the formation of reaction products of deep inelastic transfer, on the basis of which it was concluded that they can be a good method for the synthesis of new nuclei, including neutron-rich isotopes of light elements. It is very important to know how the cross section for the formation of isotopes changes as the distance from the line of $\beta$-stability increases. In [52], a method was proposed for describing the dependence of the cross section for the production of isotopes on the number of neutrons by means of the so-called $Q_{g g}$ systematics. According to this systematics, the isotope formation cross sections lie on a straight line whose slope is the same for all the isotopes of one element formed in the given reaction (Figure 7 ).

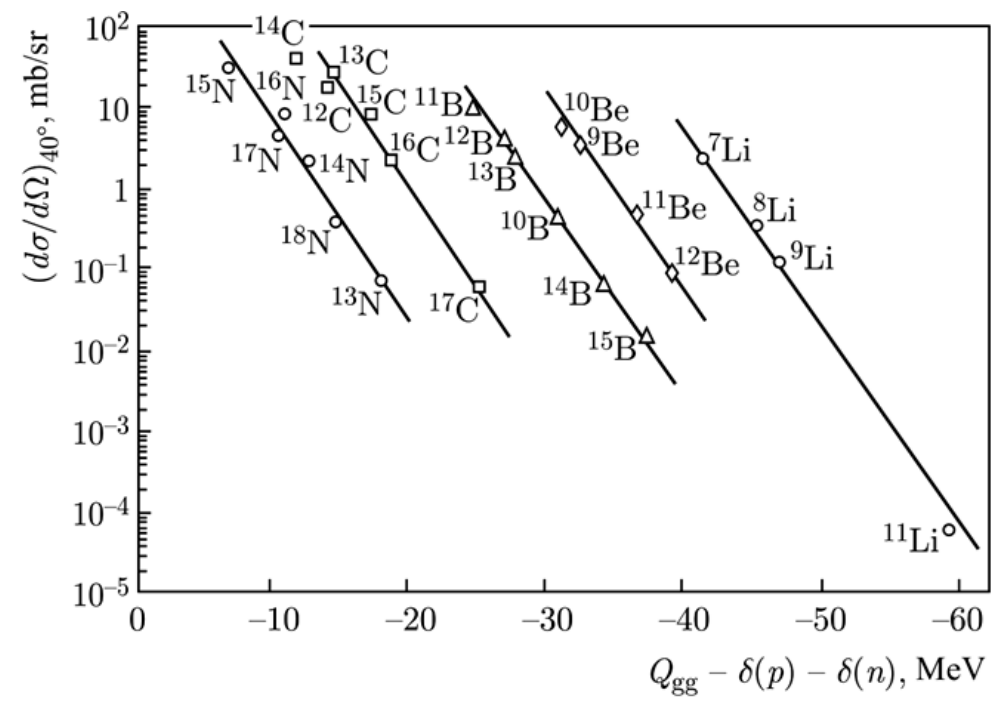

Figure 7. $Q_{g g}$-systematics of the cross sections for the formation of products of the transfer reactions in the interaction of ${ }^{232}$ Th with ${ }^{16} \mathrm{O}(137 \mathrm{MeV})[51,52]$.

Therefore, $Q_{g g}$ systematics can be used to extrapolate the cross sections for the formation of unknown isotopes. However, this is done with an accuracy determined by the accuracy of the mass values calculated using various mass formulas, since the value of $Q_{g g}$ is equal to the difference in the mass of the nuclei in the initial and exit channels, i.e., $Q_{g g}=\left(M_{1}+M_{2}\right)-\left(M_{3}+M_{4}\right)$. Given the statistical nature of the deep inelastic transfer reactions and making corrections for the pairing of protons and neutrons, the cross section for the formation of isotopes can be determined as follows [52]: 


$$
\sigma=\exp \left[\left(Q_{g g}+\Delta E_{c}+\Delta E_{r o t}-\partial(p)-\partial(n)\right) / T\right],
$$

where $\Delta E_{c}$ and $\Delta E_{\text {rot }}$ are the changes in the Coulomb and the rotational energy of the system; $\delta(p)$ and $\delta(n)$ are pairing corrections; $T$ is the temperature of the dinuclear system. This dependence is successfully used to describe the experimental regularities in the probabilities of isotope formation in the reactions of deep inelastic transfer.

However, when it comes to nuclei near the neutron stability boundary, the situation changes. For example, the cross sections for the formation of ${ }^{10} \mathrm{Li}$ and ${ }^{14}$ Be nuclei turned out to be much smaller than those predicted by the $Q_{g g}$-systematics, and for the ${ }^{10} \mathrm{He}$ nucleus only the upper boundary of its formation was obtained, two orders of magnitude lower than expected [53]. Perhaps, such deviations from the $Q_{g g}$-systematics are due to the fact that all the aforementioned nuclei are very weakly bound.

Thus, the use of the deep inelastic transfer reactions can be very efficient for the synthesis of nuclei at the nucleon stability boundary. It was shown in [21] that the use of beams of neutron-rich nuclei, such as ${ }^{48} \mathrm{Ca},{ }^{56} \mathrm{Fe}$, etc., may prove to be almost the only method for synthesis of nuclei in the region $40<\mathrm{N}<70$ near the borders of nucleon stability. Recently, the possibilities of deep inelastic transfer reactions with beams of krypton, xenon and uranium (U+U) [55] for the synthesis of heavy and superheavy nuclei are discussed. In [54], the U+U reaction was studied at a beam energy of $7.38 \mathrm{MeV} / \mathrm{A}$. Mass distributions of reaction (quasi-fission) products were obtained, from which it follows that products much heavier than uranium are formed with a relatively high probability (Figure 8). In addition, there is a certain structure in the mass distribution (bump in the region of mass 208), which, according to the authors, indicates that the shell effects are still present in such interactions. Consequently, this can be one of the methods for synthesis of nuclei near the shell numbers including superheavy nuclei with the number of neutrons 182.

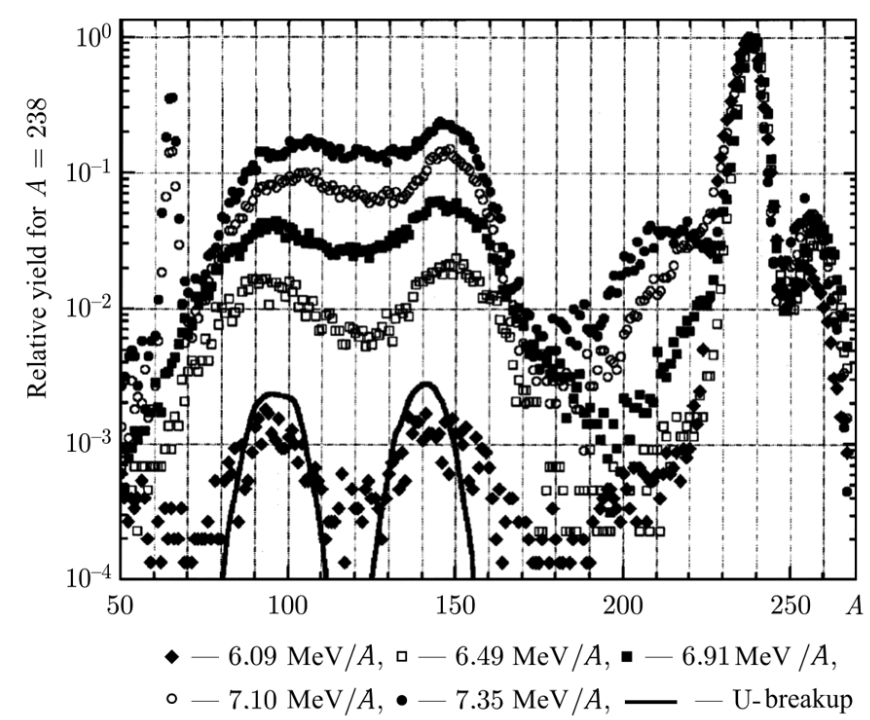

Figure 8. The mass distributions of the products of the $U+U$ reaction at different energies [54]. 


\section{Search for neutron-rich nuclei in pion absorption re- actions}

Interesting results on the synthesis of new isotopes of the lightest elements (hydrogen, helium, lithium, beryllium) were obtained in the stopped pion absorption reactions [33].

The possibility of efficient utilization of the $\pi^{-}$-meson absorption by nuclei for the formation of neutron-rich nuclear states is based on the features of this reaction: a decrease in the charge of the resulting nuclear system and a huge (on the scale of nuclear excitations) energy release.

The absorption of a pion by nuclei is a multi-nucleon process. The large energy release $\left(m_{\pi} c^{2}\right)$ leads to the formation of several fast nuclear particles, mainly neutrons, protons, deuterons, tritons and helium isotopes ${ }^{3,4} \mathrm{He}$, as well as a residue nucleus. The dominant mechanism is absorption on an intranuclear proton-neutron pair with quantum numbers of the deuteron $\left(J_{p}=1^{+}, I=0\right.$, $I_{n p}=0$ ). The theoretical description of this process, as well as absorption on a pair of nucleons with other quantum numbers, is developed quite well [33].

A distinctive property of the method using the stopped pion absorption reaction is the absence of uncertainties associated with the energy resolution and the angular divergence of the beam. The initial momentum of the system is strictly zero. The uncertainty in the energy of the initial state is only due to the difference in the binding energy of the ground (most bound) $1 s$ state and $2 p$ state, from which more than half of the pions are absorbed for the nuclei under study. For ${ }^{9} \mathrm{Be}$ and ${ }^{11} \mathrm{~B}$, this difference is 0.042 and $0.066 \mathrm{MeV}$, respectively [56].

For reaction channels in which one or two charged particles are registered, the residual nucleus will have an excess of neutrons. Table 1 shows the nuclear systems that can be investigated in two- and three-particle channels of the $\pi^{-}$meson absorption reaction by ${ }^{9}$ Be nuclei.

Table 1.

The residual nuclei formed upon the absorption of $\pi^{-}$-mesons by the ${ }^{9} \mathrm{Be}$ nuclei.

\begin{tabular}{l|l|l|l|l|l}
\hline Registered particle & \multicolumn{5}{|c}{ Inclusive measurements } \\
\hline & ${ }^{8} \mathrm{He}$ & ${ }^{7} \mathrm{He}$ & ${ }^{6} \mathrm{He}$ & ${ }^{6} \mathrm{H}$ & ${ }^{5} \mathrm{H}$ \\
\hline$p$ & ${ }^{7} \mathrm{H}$ & ${ }^{6} \mathrm{H}$ & ${ }^{5} \mathrm{H}$ & $n$ & $n$ \\
\hline$d$ & ${ }^{6} \mathrm{H}$ & ${ }^{5} \mathrm{H}$ & ${ }^{4} \mathrm{H}$ & $n$ & $n$ \\
\hline$t$ & ${ }^{5} \mathrm{H}$ & ${ }^{4} \mathrm{H}$ & ${ }^{3} \mathrm{H}$ & $n$ & $n$ \\
\hline${ }^{3} \mathrm{He}$ & $n$ & $n$ & $n$ & & \\
\hline${ }^{4} \mathrm{He}$ & $n$ & $n$ & $n$ & & \\
\hline
\end{tabular}

Table 1 demonstrates the advantage of using the stopped pion absorption reaction in the study of neutron-rich nuclei. Within one experiment, it is possible to obtain information on a wide range of neutron-rich nuclei, including the wellstudied isotopes of helium and lithium, the poorly-studied superheavy hydrogen isotopes and multineutrons, the question of whose existence remains open.

However, in addition to the advantages listed above, the proposed method also has some drawbacks. First of all, it should be noted that there are no reliable 
theoretical models describing the investigated reactions. Because of this, the physical background in the measurements, due to the many-particle reaction channels, has to be described by means of distributions over phase volumes. In the case of narrow states, this approximation does not limit the quality of the results on determining the parameters of the obtained states. It is also very difficult to study the decay products of the investigated state in the stopped pion absorption reaction. This is due to the fairly low energies of the particles formed. Because of these two reasons, in this method it is very difficult to determine the quantum numbers of the investigated state.

In this rather brief description of the possibilities for obtaining exotic nuclei, we do not pretend to the completeness of the representation of various nuclear reactions and methods for studying nuclei at the boundaries of nucleon stability. We have outlined only the main reactions and methods, which, in our opinion, are successfully used at the present time and provide a solid result. Meanwhile, since the synthesis of new nuclei is a fundamental problem of many fields, not only of nuclear physics, but also of elementary particle physics, naturally, a wide variety of beams are used to solve this problem - from gamma quanta and pions to heavy ions of high energy. For example, in Los Alamos (LAMPF), an experiment was carried out based on the method of missing masses with $\pi^{+}$-meson beams [57], in which new information was obtained on superheavy hydrogen isotopes, isotopes of helium ${ }^{6} \mathrm{He},{ }^{7} \mathrm{He},{ }^{8} \mathrm{He}$, lithium ${ }^{10} \mathrm{Li},{ }^{11} \mathrm{Li},{ }^{12} \mathrm{Li}$, and beryllium ${ }^{13} \mathrm{Be}$ [33]. In GSI (Darmstadt), a new method was used to synthesize nuclei in the fission reaction of uranium nuclei excited by a giant resonance in the interaction of these nuclei accelerated up to $300 \mathrm{MeV} / \mathrm{A}$ with lead nuclei [57]. In these experiments, among many other new nuclei, a doubly magic nucleus ${ }^{78} \mathrm{Ni}$ was discovered, which has long been tried to obtain in different experiments.

The use of beams of radioactive nuclei is an efficient method of synthesizing new nuclei and studying their properties.

Experimental studies using beams of radioactive nuclei are a new, intensively developing direction in the physics of exotic nuclei. Research in this direction is broadening, which is due to the appearance of new powerful accelerators of heavy ions of medium and intermediate energies in many countries of the world. These are the accelerator complex UNILAC-SIS-ESR and the new accelerated complex FAIR in Darmstadt (Germany), the accelerator complex GANIL-SPIRAL1 and the constructed new complex for acceleration of radioactive beams SPIRAL2 in Caen (France), the heavy-ion accelerator complex RIKEN (Japan), the superconducting cyclotron in Michigan (USA), the cyclotron heavy-ion complex with a storage ring in Lanzhou (China), the superconducting cyclotron in Catania (Italy), the cyclotron heavy-ion complex DRIBs at the G.N. Flerov Laboratory of Nuclear Reactions of JINR (Dubna, Russia). A new generation of accelerators providing the possibility of obtaining high-intensity (up to $10^{14} \mathrm{~s}^{-1}$ ) beams of charged particles from protons and helium nuclei to uranium ions, gave rise to a new stage of nuclear research using beams of radioactive nuclei. 


\section{Acknowledgements}

The article is dedicated to the memory of professor A.A. Ogloblin. The author acknowledges support from the bilateral agreement JINR/CNRS/IN2P3 (France) No. 06-75, and the Russian Foundation for Basic Research, Grant No. 17-12-01170.

\section{References}

[1] Yu.E. Penionzhkevich, Eurasian Journal of Physics and Functional Materials 4(3) (2020) 186-200.

[2] T. Hoshino et al., Nucl. Phys. A 523 (1991) 228.

[3] R. Bayer et al., Czech. J. Phys. B 31 (1981) 1273.

[4] D.V. Aleksandrov et al., JaF. 35 (1982) 277. (in Russian)

[5] P. Armbruster et al., Europhys. Lett. 4 (1988) 793.

[6] Y.T. Oganessian et al., Nucl. Phys. A 303 (1978) 259.

[7] M. Rajagopalan, T.D. Thomas, Phys. Rev. C 5 (1972) 1402.

[8] E. Duek et al., Z. Phys. A 317 (1984) 83.

[9] A.M. Poskanzer et al., Phys. Rev. Lett. 17 (1966) 1271.

[10] T.D. Thomas et al., Phys. Lett. B 27 (1968) 504.

[11] A.G. Artukh et al., Nucl. Phys. A 137 (1969) 348.

[12] O.V. Lozhkin et al., Preprint RI-168. L. (1983). (in Russian)

[13] V.V. Volkov, JeChAJa 2 (1971) 285. (in Russian)

[14] P. Auger et al., Z. Phys. A 289 (1979) 255.

[15] D.E. Greiner et al., Phys. Rev. Lett. 35 (1975) 152.

[16] T. Symons et al., Phys. Rev. Lett. 42 (1979) 40.

[17] D. Guillemaud-Mueller et al., Z. Phys. A 322 (1985) 415.

[18] O.B. Tarasov, D. Bazin, Nucl. Instr. Meth. 204 (2003) 174.

[19] J.D. Stevenson, J.P. Price, Phys. Rev. C 24 (1981) 2102.

[20] D. Guillemaud-Mueller et al., Phys. Rev. C 41 (1990) 937.

[21] D. Guillemaud-Mueller et al., Z. Phys. A 322 (1985) 415.

[22] H. Sakurai et al., Phys. Rev. C 54 (1996) R2802.

[23] H. Sakurai et al., Nucl. Phys. A 616 (1997) 311.

[24] F.M. Marques et al., Phys. Lett. B 381 (1996) 407.

[25] I. Tanihata et al., Phys. Rev. Lett. 55 (1985) 2676.

[26] A.A. Korsheninnikov et al., Phys. Lett. B 326 (1994) 31.

[27] S. Lukyanov et al., Eurasian Journal of Physics and Functional Materials 4(4) (2020) 274-280.

[28] W. Mittig et al., Preprint GANIL. (1994) 94.

[29] H.C. Britt, A.R. Quinton, Phys. Rev. 124 (1961) 877.

[30] C. Borcea et al., Nucl. Phys. A 391 (1982) 520.

[31] A.A. Ogloblin, Yu.E. Penionzhkevich, Plenum Press 8 (1989) 260.30.

[32] Yu.E. Penionzhkevich et al., Physics of Elementary Particles and Atomic

Nuclei 17(2) (1986) 165. (in Russian)

[33] Ju.B. Gurov et al., JeChAJa 40(4) (2009) 1063. (in Russian)

[34] Ju.B. Gurov et al., Izvestija RAN. Ser. fiz. 74(4) (2010) 469. (in Russian) 
[35] K. Seth, Phys. Rev. Lett. 58 (1987) 1930.

[36] S.M. Drake et al., Phys. Rev. Lett. 45 (1980) 1765.

[37] F. Naulin et al., Phys. Rev. C 25 (1982) 1074.

[38] W. von Oertzen, Nucl. Phys. A 482 (1988) 357.

[39] H.G. Bohlen, Proc. Intern. Symp. on the Structure and Reactions with Unstable Nuclei, Niigata (1991) 83.

[40] A.N. Ostrowski et al., Z. Phys. A 343 (1992) 489.

[41] A.N. Ostrowski et al., Phys. Lett. B 338 (1994) 13.

[42] I. Tanihata, Nucl. Phys. A 522 (1991) 275.

[43] H.G. Bohlen et al., Z. Phys. A 351 (1995) 7.

[44] W. von Oertzen, Nuclear Collisions from the Mean-Field into the Fragmentation Regime. CXII Corso (1991) 459.

[45] N. Anyas-Weiss et al., Phys. Rep. 12 (1974) 201.

[46] H. Lenske et al., Phys. Rev. Lett. 62 (1989) 1457.

[47] I.J. Thompson, Comp. Phys. Com. 7 (1988) 167.

[48] V.V. Volkov, JeChAJa 2 (1971) 285. (in Russian)

[49] Ja. Vil'chinskij et al., JaF. 5 (1967) 942. (in Russian)

[50] D. Guerreau et al., Z. Phys. A 295 (1980) 105.

[51] A.G. Artukh et al., Nucl. Phys. A 160 (1971) 511.

[52] V.V. Volkov, Phys. Rep. 44 (1978) 93.

[53] Ju.C. Oganesjan et al., Pis'ma v ZhJeTF 36(3) (1982) 104. (in Russian)

[54] A.C. Villari et al., Phys. Lett. B 268 (1991) 345.

[55] V.I. Zagrebaev, Phys. Rev. C 67 (2003) 061601.

[56] I. Talmi, I. Unna, Phys. Rev. Lett. 4 (1960) 469.

[57] M. Bernas et al., Nucl. Phys. A 616 (1997) 352. 